\title{
Photostress Recovery Time Among Welders in Owerri Municipal Council, Southeast, Nigeria.
}

\author{
Augustine U. Akujobi ${ }^{1}$, Gift O. Uzomah ${ }^{2}$, Oforbuike O. Ike ${ }^{3}$, John C. Ekenze', \\ Jacqueline E. Obioma-Elemba ${ }^{1}$, Okechukwu A. Akogu ${ }^{1}$

\footnotetext{
${ }^{1}$ Department of Optometry, Imo State University, Owerri, Nigeria.

${ }^{2}$ Department of Ophthalmology, Nnamdi Azikiwe University Teaching Hospital, Nnewi, Nigeria.
} \\ ${ }^{3}$ Department of Optometry, Bayero University, Kano, Nigeria.
}

Corresponding author: Augustine U. Akujobi $\mid$ Email: au.akujobi@imsu.edu.ng | Phone: +2348033727967

\begin{abstract}
Purpose: The photostress recovery time (PSRT) scores of welders were measured based on age, workexperience and welding-method.

Methods: A quasi-experimental study was conducted among 50 purposively sampled welders, who had clear ocular media, healthy fundi and visual acuities of $6 / 6$ or better in the tested eyes. The pre-bleach distant visual acuity of the right eye was determined with the Snellen's visual acuity chart, while direct ophthalmoscopy was done to rule out media opacities, retinopathies and maculopathies. The right eye was dazzled for 10 seconds with the light of a direct ophthalmoscope held at $2 \mathrm{~cm}$ from the eye. The time required in seconds to read at least, three optotypes on the line directly above the baseline acuity was recorded as the PSRT.
\end{abstract}

Results: The 22-31 years age group recorded the least mean PSRT (16 \pm 8.8 seconds), followed by the 32-41 age group (19.9 \pm 6.8 seconds), while the $42-51$ years category had the highest mean PSRT $(31.4 \pm 10.5$ seconds). Age associated significantly $(\mathrm{p}<0.05)$ with mean PSRT. Welders within the 1-12 years' work experience had the least mean PSRT (15.9 \pm 8.1 seconds) while those within the 25-36 years recorded the highest mean PSRT (33.4 \pm 9.3 seconds). The observed difference in mean PSRT with years of work experience was statistically significant $(p<0.05)$. Gas welders had significantly $(p<0.05)$ higher mean PSRT (26.6 \pm 9.9 secs) than electric arc welders (16.6 $\pm 9.2 \mathrm{secs})$.

Conclusion: Gas welding, increasing age and work-experience were found to be predictive markers of elevated photostress recovery time (PSRT) among welders. This underscores the need for eye-care practitioners to routinize PSRT tests in the ocular assessment of welders..

Keywords: Photostress recovery time (PSRT); electric-arc; gas welding; welders; age.

\section{Introduction}

Welding processes emit ultraviolet radiations (200$400 \mathrm{~nm})$, visible light $(400-700 \mathrm{~nm})$ and infrared radiations (700-1400nm). Gas welding generates visible light and infrared radiations, while electric arc welding in addition to these, produces ultraviolet radiations ${ }^{1}$. 
Although these radiations are absorbed by different ocular tissues and harmful to the eyes to different degrees, visible light is the most noticeable to the welder ${ }^{1}$. Visible light falls within the range of $400-700 \mathrm{~nm}$ and wavelengths outside this scope are invisible to the human eye. Ultraviolet and far infrared (IR) radiations are absorbed by the cornea and lens, whereas visible light and near infrared penetrate to the retina, where they create some biochemical phototoxic effects ${ }^{2,3}$. Depending on the intensity and duration of exposure, the phototoxic effects caused by these radiations can be irreversible and sight threatening ${ }^{4}$.

Within the visible light spectrum, high energy short wave blue light between 415 and $455 \mathrm{~nm}$ is the most harmful ${ }^{5}$. An experimental study ${ }^{6}$ on blue lightinduced oxidative stress injury on rabbit retinas showed that the rabbit retinas, after 24 hours of blue light irradiation, had become disordered in the inner and outer segments of the photoreceptor cells when compared with the normal control group. Other phototoxic effects of blue light on the retina include retinal (macular) degeneration, damage of blood retinal barrier function and oxidative stress injury of the retina ${ }^{5}$. In both electric arc and gas welding, blue light is emitted at very high temperatures which are predominantly responsible for photoretinopathy among welders. It has been argued that the mechanism of light-induced injury associated with arc welding is not thermal because the temperature rise in the retina is estimated to be insufficient to cause a burn, therefore, the injury mechanism is considered to be photochemical ${ }^{7}$. The hazard of blue light is generally measured by effective bluelight radiance ${ }^{8}$.

The retina contains cone and rod photoreceptors. The rods contain visual pigments known as rhodopsin. Rhodopsin is made up of opsin and 11-cisretinal. Upon exposure to visible light, rhodopsin undergoes some biochemical reactions where the 11-cis-retinal is converted to all-trans-retinal in a process known as bleaching. When illumination is extinguished, the all-trans-retinal released during bleaching is either stored or reconverted to 11-cisretinal and transported back to the rhodopsin in the rods in a process known as re-synthesis or recycling $9,10,11$. The bleaching and re-synthesis of rhodopsin are clinically significant and provide a quantitative index of macular function in photostress recovery time (PSRT) tests.

Photostress recovery time is the time in seconds between bleaching and re-synthesis. Clinically, it is the time required in seconds for the macula to return to its normal level of function after being exposed to a bright light source ${ }^{12}$. Although many studies have put the normal PSRT at $\leq 50$ seconds $13,14,15,16$, a study ${ }^{17}$ opined that the upper limit of the normal range may vary to as much as 80 seconds as a result of differences in photostress stimulus

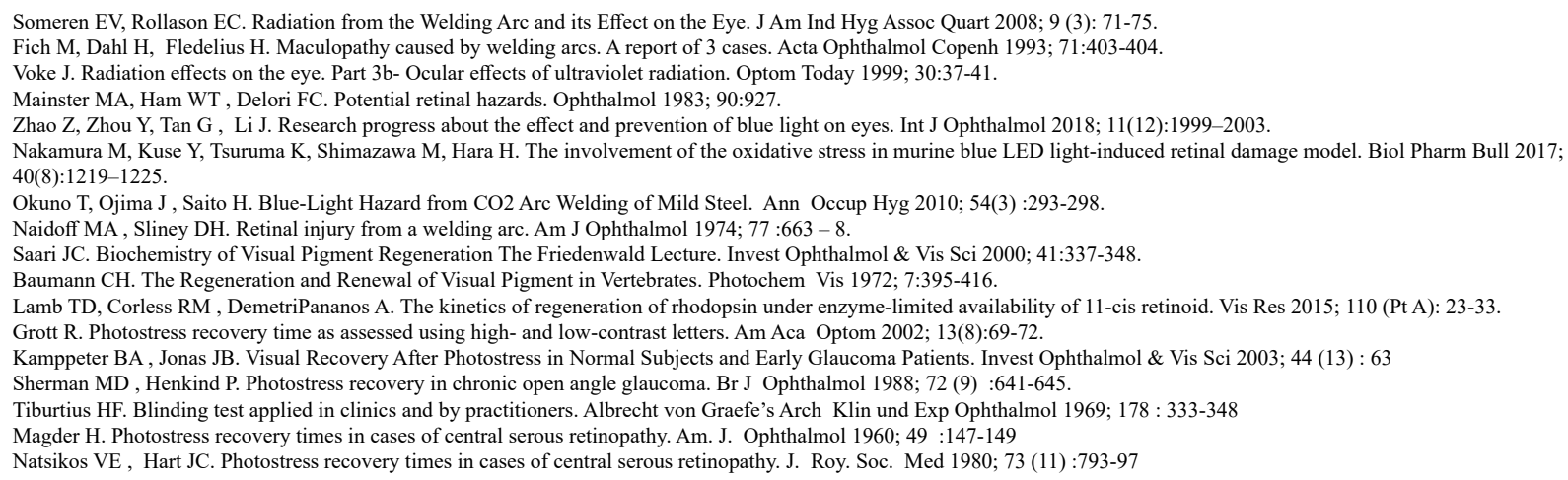


and technique employed. However, the study ${ }^{17}$ was criticized for being obsolete and uncertain about the extent of healing while evaluating the mean PSRTs in central serous retinopathy at the acute, resolving and resolved stages.

Lack of standardization, with respect to fixation loss of the bleaching light, definition of target clarity, timing accuracy and dazzling stimulus, has been identified as an underpinning challenge in PSRT assessment. However, Glaser et al. ${ }^{18}$ used a penlight to dazzle the eye and found a corresponding PSRT score with another study ${ }^{19}$ which used high intensity light flash system as a photostress stimulus. Omokhua \& George $^{20}$ in a study conducted in Nigeria, observed a mean PSRT range of 10.34seconds to 46.98 seconds for an age range of 11 years to 70 years using a penlight as dazzling stimulus. Therefore, the need for standardized test protocols cannot be over-emphasized.

Despite the hazards posed by blue light and other radiations emitted during welding, priority attention has not been given to the evaluation of retinal (macular) functions of welders using the photostress recovery time (PSRT) test. Within the purview of ergonomics, it is imperative that welders become knowledgeable in the hazardous effects of these radiations on the retina, especially on macular functions; and the usefulness of the PSRT in periodic macular evaluation. The growing knowledge and medical evidences corroborating the admissibility and reliability of the PSRT as a costeffective diagnostic test, capable of distinguishing between macular and optic nerve lesions, challenge researchers to communicate the science of PSRT to welders.

Sequel to systematic searches, no study was found to have investigated the age and work-experience patterns of PSRT among welders, neither was there a comparative study conducted on the PSRT of electric arc and gas welders. Thus, the present study, to the best of the researchers' knowledge, may be the first to examine the age and workexperience patterns of PSRT and most importantly, to quantitatively compare the PSRT scores of electric arc and gas welders.

\section{Methods}

\section{Study design and study area}

Quasi-experimental study design was used to measure the photostress recovery time of gas welders and electric arc welders in Owerri, Imo State, Nigeria.

\section{Sample size and sampling technique}

Fifty (50) welders, (25 gas welders and 25 electric arc welders) were purposively recruited from Owerri, Imo State, Nigeria for the study. The selected welders who consented to participate in the study were invited to the Imo State University Optometry Clinic, Owerri, Nigeria for the study. The sample size was determined using the Taro Yamane's formula for determination of minimum sample size, considering the number of welders in the study area and $95 \%$ confidence interval.

Natsikos VE, Hart JC. Photostress recovery times in cases of central serous retinopathy. J. Roy. Soc. Med 1980; 73 (11) :793-97

Glaser JS, Sarino KD, McDonald, SA, Knighton RW. Photostress recovery test in the clinical assessment of visual function. Am. J. Ophthalmol 1979; $83: 255-60$.

Severine SI, Tour RI, Kershaw RH. Macular function and the Photostress Test 2. Arch Ophthalmol 1967; 77:163-7.

Omokhua PO, George OG. Photostress recovery time among Nigerians. J. Nig Optom Assoc 2010; 16 :16-20. 


\section{Ethical clearance}

The study complied with the protocols of Helsinki Declaration on Human Experiments. The study was approved by the Research/Thesis Committee of the Department of Optometry, Imo State University, Owerri, Nigeria. Written informed consents of the participants were also obtained.

\section{Inclusion criteria}

Welders 18 years and above, who had clear ocular media, healthy fundi and visual acuities of $6 / 6$ or better in the tested eyes were recruited into the study.

\section{Exclusion criteria}

Participants who had either media opacities, retinopathies, maculopathies or were hypersensitive to the photo stressor were excluded from the study. Welders below the age of 18 and those who did not give their consent were also excluded.

\section{Procedure for data collection}

Each participant who satisfied the inclusion criteria was given 5 minutes to adapt to the ambient illumination of the clinic. While the left eye was under occlusion with a Dukal Single Ended Paddle Occluder, the entry distant visual acuity of the right eye was measured using the Snellen's visual acuity chart. Subsequently, direct ophthalmoscopy was performed on the right eye to rule out media opacity, retinopathy or maculopathy. To dazzle the right eye, the light source of the direct ophthalmoscope was held at $2 \mathrm{~cm}$ along its visual axis for 10 seconds with the left eye occluded. At 10 seconds, the dazzling stimulus was withdrawn from the right eye, while the left eye remained under occlusion and the participant was instructed to read the Snellen's optotypes with the right eye. The time required in seconds for the participant to read at least, 3 optotypes on the acuity line directly above the prebleached level was measured by a stopwatch and recorded as the photostress recovery time (PSRT) score.

\section{Statistical analysis}

Age and work-experience distributions of mean PSRT were analyzed with the One-way Analysis of Variance (ANOVA), while the welding-type distribution of mean PSRT was analyzed using the Two Sample Z-test, both at 0.05 level of significance. $\mathrm{P}<0.05$ was considered statistically significant.

\section{Results}

The 22-31 years group presented the highest number of participants $20(40 \%)$, followed by the 32-41 age category with $16(32 \%)$ participants, while the 42 51 years group had the least number of participants 14 (28\%). The 22-31 years category had the least mean PSRT (16 \pm 8.8 seconds), followed by the $32-41$ years group (19.9 \pm 6.8 seconds) and the 42 51 years group with the highest mean PSRT of $31.4 \pm 10.5$ seconds (Table 1 ). 
Table 1: Age distribution and mean photostress recovery time (PSRT) of welders

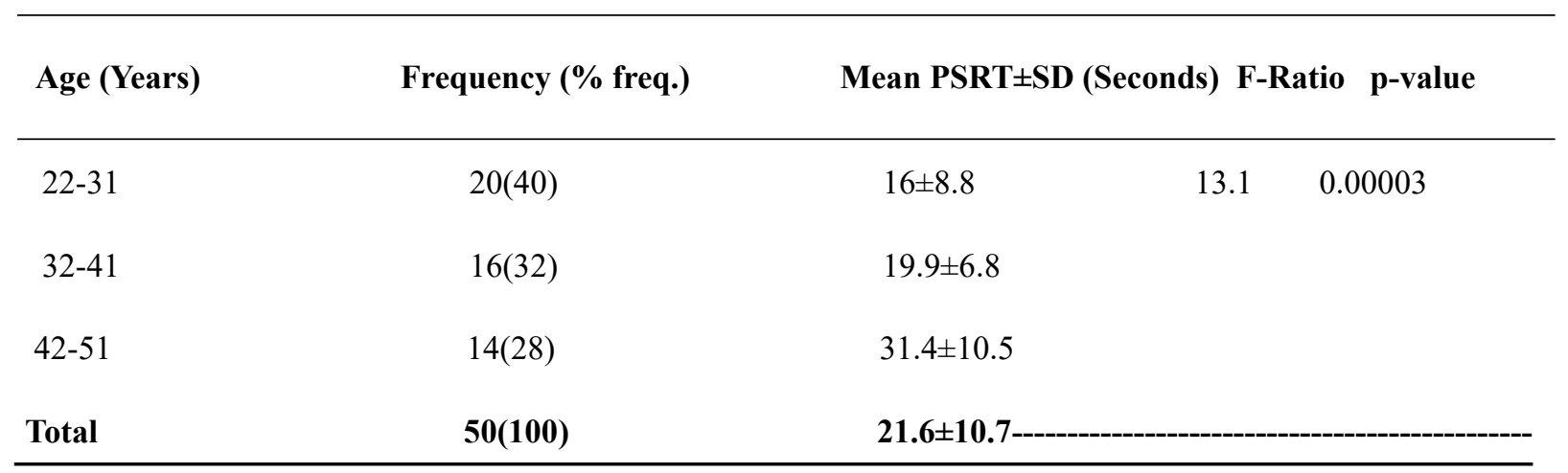

Welders who had worked for 1-12 years recorded the least mean PSRT (15.9 \pm 8.1 seconds), followed by those within the 13-24 years' work-experience (22.7 \pm 8.6 seconds), while welders within the 25-36 years' work-experience had the highest mean PSRT (33.4 \pm 9.3 seconds). The overall mean PSRT of welders was observed to be $21.6 \pm 10.7$ seconds (Table 2).

Table 2: Distribution of mean photostress recovery time (PSRT) of welders based on years of workexperience

\begin{tabular}{lcccc}
\hline Work experience (Years) & Frequency (\% freq.) & Mean PSRT \pm SD (Seconds) & F-Ratio & p-value \\
\hline $1-12$ & $24(48)$ & $15.9 \pm 8.1$ & 15 & 0.00001 \\
$13-24$ & $16(32)$ & $22.7 \pm 8.6$ & \\
$25-36$ & $10(20)$ & $33.4 \pm 9.3$ & \\
Total & $\mathbf{5 0 ( 1 0 0 )}$ & $\mathbf{2 1 . 6 \pm 1 0 . 7 - - - - - - - - - - - - - - - - - - - - - - - - - - - - - - - - - ~}$ \\
\hline
\end{tabular}

Electric arc welders had a mean PSRT of $16.6 \pm 9.2$ seconds, while gas welders recorded a mean PSRT of 26.6 \pm 9.9 seconds.

Table 3: Distribution of mean photostress recovery time (PSRT) among electric arc and gas welders

\begin{tabular}{lllll}
\hline Method of welding & Frequency (\% freq.) & Mean PSRT \pm SD (Seconds) & Z-score & p-value \\
\hline Electric arc & $25(50 \%)$ & $16.6 \pm 9.2$ & 3.694 & 0.0002 \\
Gas & $25(50 \%)$ & $26.6 \pm 9.9$ & \\
Total & $\mathbf{5 0}(\mathbf{1 0 0})$ & $\mathbf{2 1 . 6 \pm 1 0 . 7}$ & & \\
\hline
\end{tabular}




\section{Discussion}

The welders within the age group of 42-51years recorded the highest mean PSRT $(31.4 \pm 10.5$ seconds) while those in 22-31 years category had the least mean PSRT (16 \pm 8.8 seconds). The overall mean PSRT of the welders was found to be $21.6 \pm 10.7$ seconds. The results from this study, demonstrated, along with previous studies ${ }^{20,21,22}$, a statistically significant $(p<0.05)$ relationship between age and PSRT. In addition, the mean PSRTs of all age groups examined were found to be within normal range ( $\leq 50$ seconds), representative of the healthy fundi (macular) of the study participants.

It is known that increased optical density of the aged crystalline lens and pupillary miosis contribute to older adults' bleach-resynthesis cycle, but they are not primarily responsible for increased PSRT scores ${ }^{23,24}$. Although little is known about the neural contribution to this deficit, it has been demonstrated that with advancing age, even up to the seventh decade of life, the density of rod photoreceptors decreases dramatically in the perimacular region ${ }^{25,26}$. Conversely, discourses abound which have argued that scotopic sensitivity loss in older adults is observed in peripheral retinal areas where there is negligible rod loss and is not accentuated in the areas of heightened rod $\operatorname{loss}^{24}$ and that there are sufficient evidences demonstrating little changes in the amount of rod photopigment (rhodopsin) throughout adulthood ${ }^{27,28,29}$. These connote that rod loss cannot account for older adults' sensitivity impairment in the dark. But recently, it has been alternatively hypothesized that the scotopic sensitivity loss associated with aging is as a result of the disruption of the visual cycle by age $^{30,31}$.

Welders who had worked for 1-12 years had the least mean PSRT (15.9 $\pm 8.1 \mathrm{secs})$, while those within the 13-24 years on-the-job experience recorded a mean PSRT of $22.7 \pm 8.6$ seconds. Those who had worked for 25-36 years were observed to have the highest mean PSRT (33.4 $\pm 9.3 \mathrm{secs})$. The distribution of mean PSRTs varied directly and significantly $(p<0.05)$ with work-experience. Age and workexperience exhibited similar PSRT patterns, thus, the mean PSRT presented by different workexperiences may not be unconnected with the age of the participants. It is definite that age has a strong correlation with work-experience; hence, there may have been a likely overlap between age and workexperience.

Gas welders had significantly $(\mathrm{p}<0.05)$ higher mean PSRT $(26.6 \pm 9.9 \mathrm{secs})$ than electric arc welders (16.6 \pm 9.2 secs). On the other hand, it is known that electric arc exhibits higher effective blue light radiance than gas, hence, generates more heat $\left(10,000-20,000^{\circ} \mathrm{F}\right)$ when compared with gas flames $\left(4000-6300^{\circ} \mathrm{F}\right)$. The implication is that the blue light of electric arc is more harmful than that of gas flames and ought to demonstrate higher mean

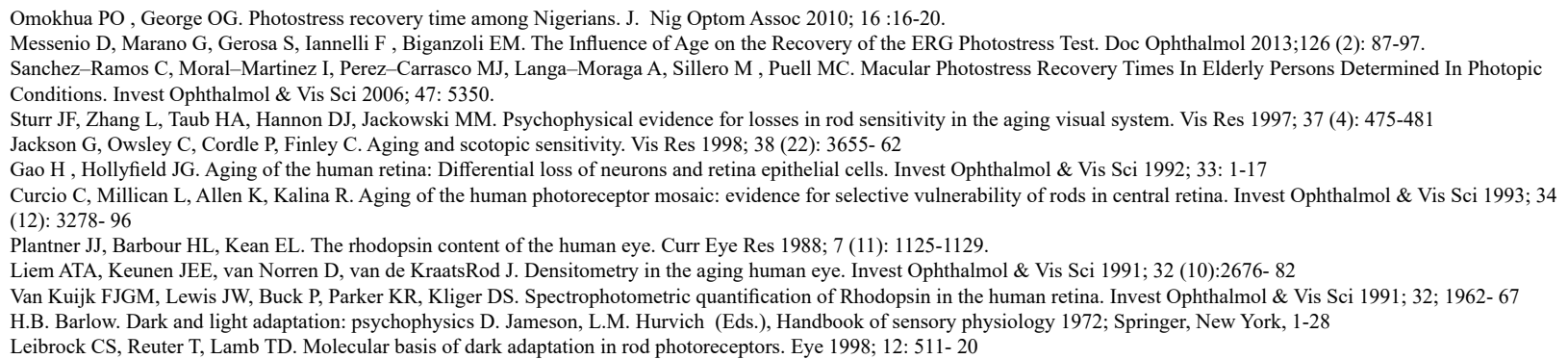


PSRT than gas welding. However, the present study did not typify this hypothesis. In addition to the temperature of the irradiance, it has been opined that other factors such as exposure duration ${ }^{32}$ and type of Personal Protective Equipment (PPE) also account for the level of blue light-associated harm suffered by the retina. In the present study, the aberrant PSRT scores reported may be characterized by these unverified parameters. Furthermore, it is also possible that more young adults engaged in electric arc welding than the older population which explains the skew towards lower mean PSRT among electric arc welders. The mean PSRTs of electric arc and gas welders were remarkably within normal PSRT range because the sampled welders had apparently healthy maculae.

A study ${ }^{33}$ had shown that visual functions, glare discomfort and PSRT were significantly improved when activated photochromic lenses were worn as against clear lenses. The magnitude of relief and protection provided by these lenses deserve further investigations in order to ascertain their viability as PPEs for welders. In addition, the present study did not provide isolated data for the work-duration and age-specific patterns of PSRT of electric arc and gas welders, thus, further studies in this area are expedient. Also, the present study did not have a control, therefore a true experimental study is recommended in order to draw more conclusions on the postulates of the study.

\section{Conclusion}

The observed relationship between mean PSRT scores, age of welders, work-experience in years and welding-methods, underscores the need for eye-care practitioners to incorporate PSRT tests in routine eye examination of welders and to educate welders on the importance of regular PSRT checks and macular function evaluations.

\section{Acknowledgement}

The authors are grateful to the Department of Optometry, Imo State University, Owerri, Nigeria for granting approval for the use of the Optometry Clinic.

\section{Declaration of conflict of interest}

The authors declare that there is no conflict of interest. 